\title{
Changing the face of Portuguese Family Medicine
}

\section{An overview of the recent history of family medicine in Portugal}

\author{
JAIME CORREIA DE SOUSA, MD, MSc* LUÍS PISCO, MD**
}

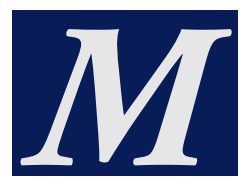

ost family physicians are knowledgeable about the major health systems existing in Europe, as this has been the subject of many publications in the medical press. When we decided to produce a special issue of the Portuguese Journal of General Practice it seemed likely that the profile and recent history of Portuguese Family Medicine would be less known to non-Portuguese readers and, therefore, an article on Family Medicine and the Health System in Portugal seemed appropriate.

In 1974, democracy was restored in Portugal. As with many other institutions and organisations in the country, health services initiated a period of reforms aiming at providing health care for all citizens. ${ }^{1,2}$ These reforms affected both hospitals and ambulatory services and produced significant changes in mortality rates and health status with a great

*Famíly Physician. Lecturer in the School of Health Sciences in Minho University. Chief-Editor of the Portuguese Journal of General Practice.

**Famiily Physician. President of the Portuguese Association of General Practitioners. Chairman of the Taskforce for the reform of Primary Health Care. impact on Portuguese society. ${ }^{3}$

This paper intends to give a picture of the present situation and describe the importance and position accomplished by family medicine, its strengths and weaknesses, and the opportunities created by the newly proposed reforms.

\section{The Portuguese Health System}

\section{Organisation}

Portugal has a National Health Service (NHS), since 1979. All Portuguese citizens or foreign residents have general health coverage. Most physicians are employees of the NHS. However, a considerable number of doctors can also work in the private sector. Roughly ninety per cent of people are registered with family physicians in the community health centres of the NHS. ${ }^{4}$ Others have alternative health insurances or health coverage plans through employers or companies.

Within the NHS, access to secondary and tertiary care is through referrals from family doctors. However, in emergency services, private practice and some health plans outside the NHS, patients can go directly to specialists.

In the early 1970s, Portugal was one of the first European countries to adopt an integrated approach in primary health care through the development of an impressive health centre network. Currently there are about 350 health centres and almost 2,000 health stations covering most of the national territory.

This network, along with better maternity care at hospital level, have been deemed responsible for very significant advances in health status: in the late 1960s the Portuguese child mortality rate was in the 60 per 1,000 range, by far the highest among the other 15 European Union countries. ${ }^{5}$ Thirty years later it was 5,0 per 1,000 . This is a better rate than in Greece, Netherlands, Ireland, Luxembourg and the United Kingdom, and similar to Belgium, ranking ninth in the EU. ${ }^{6}$

Family doctors are expected to have patient lists of 1,500 people, on average; however, due to a manpower shortage in the profession many family physicians have larger patient lists. Some of this shortage is caused by the uneven distribution of family doctors, thus causing irregular list sizes; in the inner cities, some doctors might have fewer patients, but in the suburban areas and some parts of the countryside the figures are often higher.

Family physicians are responsible for a wide range of activities, including general medical care for the adult population, prenatal care, child care, women's health, family planning, first aid, certification of incapacity to work, home visits, preventive services, including immunisation and screening for breast and

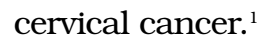

Manpower, though, is insufficient. A substantial number of Portuguese are waiting for a place on a 
family doctor's patient list, the number of family doctors being below the OECD average. ${ }^{7}$ Family doctors, those specialized in family medicine, account for $29.5 \%$ of the total number of doctors in the NHS; $42.5 \%$ are hospital doctors and $2 \%$ are public health doctors; 44 per cent are female; 24.2 per cent are postgraduate residents; there were 3.2 physicians per 1,000 population in $2000 .^{1}$

The NHS owns most of the hospitals and health centres. A smaller number of hospitals belongs to charities and have established partnerships with the NHS. Private hospitals and clinics are not part of the NHS, but have contracts with private health plans and insurance. Occasionally, private hospitals are used by the public sector to help reduce surgery waiting lists.

The cooperation between primary and secondary care is inadequate; several attempts have been made to improve it, with limited results. The disconnection concerns both administrative and clinical procedures and it results in a considerable waste of resources, due to the duplication of tasks, repetition of investigations, and the inability to provide the follow-up care the patient requires after hospital treatment because the family physician does not always receive a report of the outcomes and diagnosis for follow-up.

\section{Health care financing and expenditure}

The NHS is predominantly funded through general taxation. Employer and employee contributions represent the main funding sources of the other health subsystems. In addition, direct payments by the patient and voluntary health insurance premiums account for a considerable proportion of funding. The public hospital sector accounts for about half of the NHS expenditure. ${ }^{7}$ Primary health care centres are financed by the NHS through the regional health administrations and do not have financial or administrative autonomy. In 2002 Portugal's expenditure on health amounted to $9.3 \%$ of GDP.

\section{Payment system}

Within the NHS, most physicians are paid on a salary basis, both in hospitals and health centres. There is a complex payment system according to seniority, the contract and extra duty hours; consequently salaries have a wide variation. Recent developments have introduced an innovative form of payment for family physicians. In the private sector the majority of doctors are paid on a fee for service basis.

\section{family Medicine in Portugal}

Modern Portuguese Family Medicine was officially born in 1982. The health care model prior to the setting of the new career was based on a unstructured combination of a considerable number of public ambulatory stations for employees, a small number of community health centres essentially directed towards preventive activities and private practices mainly concentrated in the bigger cities. There was no requirement for a specific training, and any doctor wishing to establish a practice was allowed to be referred to as "general practitioner".

Since its creation, modern Portuguese Family Medicine grew with its eyes and ears focused on European and North American family medicine although with its feet firmly planted in the Portuguese reality. The development of the profession was strongly influenced by the experien- ces and the successes achieved in countries where family medicine had been established for a long time and where it already enjoyed considerable prestige. ${ }^{8}$

In order to understand some of the strengths and weakness of Portuguese Family Medicine, it is important to recall that all of the thinking and conceptual models of primary health care at the time of the appearance of the profession in Portugal were strongly influenced by the WHO recommendations and the concepts that emerged from the Alma-Ata conference in $1978 .^{9,10}$ The model that was developed after $1980,{ }^{11,12}$ despite strong influence by the primary health care systems based on family medicine, always had great difficulty in freeing itself from that heavy community orientation.

One can trace the origin of foreign influence on the development of a family medicine culture in Portugal in several areas, ranging from the origin and launching of the profession, to specific training, to the model of professional organisation and to the emerging of undergraduate training and to research.

Some European models influenced Portuguese family medicine at its very beginning. Portuguese Family Medicine owes a lot to the early cooperation with the British Royal College of General Practitioners (RCGP) and the Oslo University Institute of General Practice. The report produced by the participants in the seminars held in 1979 at the Portuguese National School of Public Health, on "The Role of the GP in Primary Health Care" would be designated as the "Horder Report" ${ }^{13,14}$ and was a landmark for Family Medicine in Portugal. It allowed the first General Practitioners of the new era to know that they had a reference document and to feel that they belon- 
ged to a developing international movement, supported by their European colleagues. The contribution given by the RCGP was decisive. The Portuguese family doctors took their inspiration not only from the RCGP in the format of the professional organisation they wanted to develop, but also the support from British colleagues was constant from the beginning in research and in the creating of contact networks.

The Norwegian cooperation with the north of Portugal, ${ }^{15}$ both in the establishment of several health centres, and in contact and cooperation between several health professionals, doctors and nurses and their Norwegian peers, was achieved through the NORAD (Norwegian Agency for International Development) programme.

Other health systems with very different characteristics served over time as reference models in the development of a culture of family medicine. The two North-American models (from Canada and the United States) share a philosophy of family medicine which came to decisively influence the family orientation of Portuguese general practice.

It would be unfair to forget the modest but solid influence of Dutch family medicine, particularly on the level of research and on quality assurance. There was also exchange of ideas and experiences with Spain, which went through parallel and almost contemporary paths, which greatly contributed towards enriching Portuguese family medicine.

\section{Family Medicine Organisation}

The Portuguese Association of General Practitioners (APMCG), ${ }^{16}$ the professional organization of Portuguese family medicine, was structured both as a scientific and academic society and a professional organisa- tion; since the creation of family medicine in Portugal, the APMCG has distinguished itself through its support for the birth and development of modern family practice.

The Portuguese Association of General Practitioners led a constant struggle for professional quality and development of the profession, focusing on vocational training, undergraduate teaching, professional competence, specialty recognition, research development, practice organisation, team work, communication skills, and patient centred practice, among many other issues.

\section{Specific Training}

Foreign influence was also decisive in the development of the model for specific training in Family Medicine in Portugal. The European and North American countries with which we maintained relations already possessed well-organised structures for specific training, well designed and tested programmes, and vast experience in pedagogical methods. Observing and learning from these experiences was fundamental for the building of training programmes in Portugal. ${ }^{17,18}$ It was the area in which foreign influence was most significant. ${ }^{19-21}$ In the early stages, many family doctors had the opportunity to participate in training courses held in other countries. They were supported by the European organisations that made places and resources available in order to support our development. This effort would decisively mark the content and format of the General Practice Vocational Training Programme and would contribute towards the acknowledged quality of these programmes in Portugal.

Between 1981 and 1986 from all the doctors entering the new GP career, eighty-five per cent did not have specific training in general practice. Only ten per cent completed a threeyear vocational training programme. This training became mandatory after 1987. Special in-service specific training programmes were implemented for those GPs who had not had any vocational training and had been in general practice for at least five years.

In 1982 the College of General Practice was founded as the accreditation board for specialisation in general practice/family medicine as a body within the Portuguese Medical Association. Its main role is to develop the curriculum and the structure of the training programme and to participate in the final assessment of trainees.

The residency training programmes are organised and managed by the three Regional Vocational Training Coordination structures, which are appointed by and operate within the NHS; training programmes are based on the practice of family medicine in a health centre. Each doctor in training (trainee) is assigned to an accredited family physician trainer that is responsible for the tutoring. Hospital and short courses are additional elements of the training process.

The programme is based on a three-year schedule. A four-year programme has been proposed by many professional organisations but has met with considerable resistance from officials inside the NHS.

The assessment of trainees consists of a combination of methods: trainees must perform certain assigned tasks; there are assessment interviews after each training rotation; there is a yearly evaluation and a final three-day exam with a board of five family physicians. A Residency Handbook with aims, general contents, specific targets and eva- 
luation guidelines, which guides trainees and trainers throughout the training programme, supports the entire process. ${ }^{4}$

\section{The Establishment of Undergraduate Training}

The resurgence of family medicine in Portugal has created a new goal of developing undergraduate training in family medicine in medical schools.

By observing what took place in other countries, the pioneers of modern Portuguese family medicine discovered early the strategic importance of such a measure as of family medicine can only mature when its scientific credibility is supported by teaching and research connected to medical faculties.

The realisation of this aspiration, for which international influence was decisive, was only achieved after 1984 in Porto, with the creation of the first Department of General Practice (although teaching of the subject only began in 1989) and with the beginning of teaching in General Practice and Community Medicine in Lisbon in $1987 .{ }^{22}$ Today, there are seven medical schools in Portugal all of which teach family medicine either in a Community Medicine or in a Family Practice department, some of which have a significant number of weeks of practical training with clinical tutors in the health centres.

\section{Individuals and the Informal Contact Networks}

More than institutional relations, it was the people and the informal networks that provided opportunities for exchange of ideas and experiences abroad, thus providing the foundation for the development of the discipline. The whole process of networking in the most diverse areas counted on many individual contributions. Portuguese family physicians either participate actively in international organisations like UEMO (European Union of General Practitioners), WONCA (World Organisation of Family Doctors) and its associated organisations, EGPRW (research), EQUIP (quality), EURACT (teaching), EUROPREV (prevention), or have established personal or institutional relationships with family medicine colleges and academic institutions from other countries.

\section{Innovation and reform}

Despite the remarkable achievements in health policy in Portugal, many challenges remain. In comparison to other OECD countries, expenditure of the Portuguese health care sector is characterized by a high level of resources relative to the GDP, a low level of public per capita expenditure and high levels of pharmaceutical expenditure. ${ }^{23}$

According to the OECD, the results in some areas of health policy, such as immunisation policy, primary health care, and diabetes care, have seen significant achievements. But, despite these developments, the health system still faces a number of challenges: health inequities, the high level of private expenditure, and a poor coordination between primary and secondary health care. These challenges still present important obstacles, and have structural and cultural limitations. Most of them come from the bureaucratic structure that constrains family medicine in the National Health Service. Many health care reforms have been legislated, but never completely implemented. The health centres are a cultural, technical and institutional heritage which is not only important to preserve but also to modernise and develop, since they continue to be one of the most efficient and accessible means to protect and promote the population's health.

Many Portuguese family physicians are aware of the limitations and needs of such health structures, the result of years of lack of investment. They realise the chronic lack of human resources, of which the family doctors are only the most visible. There is also a shortage of family nurses, nutritionists, psychologists, social assistants, oral hygienists and consultants.

There are good international examples of the advantages of cooperation between the health professions and the main objective is to bring different professionals to work together to strengthen primary health care. ${ }^{24-26}$

Among the negative aspects of Portuguese family medicine are the very rigid, civil service-driven system which is averse to change, a reversed distribution of health professionals, aging professionals with shortage of replacement and the professionals' geographic distribution out of pace with demographic changes. Primary care is often seen as second-rate care and there has been a salary based payment system, with little or no incentives.

In an attempt to change the face of family practice in Portugal, an Experimental Payment System (EPS) for family physicians was initiated in 1999. Twenty groups of doctors, nurses and receptionists have accepted the experimental use of payment incentives. ${ }^{27}$ In the trial model, the salaries were adjusted for patient characteristics, doctors' list si$\mathrm{ze}$, and the length and nature of doctors' work..$^{28}$ Despite its name, this is more than just a payment system. The groups had a commitment to organise primary health care for a group of people ranging from 6,000 
to 16,000 and to develop strategies that increase autonomy, strengthen teamwork and produce better health outcomes. In 2005 the health ministry conducted an external audit comparing the groups under EPS with a sample of random doctors in the "old" system. The results confirmed that the groups had larger patient lists per physician and were able to perform better in most of the health indicators with lower expenses, including less use of medication, investigations and referrals.

As a result of the success of the EPS, the present Government has decided to continue the reform of the primary health sector allowing more family physicians to start new group practices and reforming the health centres. A special task force ${ }^{29}$ (the Taskforce for the reform of Primary Health Care - MCSP) has been created to give logistical support to the groups wishing to organise in the new contract.

In the Government's program it is stated that primary health care is the Health System's central support and that Health Centres are the institutional base of primary health care. To make this vision come true, it was necessary to change the current primary health care scene, making it more accessible, more responsive to needs and more efficient, in response to the expectations of both citizens and professionals, giving better care and getting better health.

Restructuring is in progress in eight major areas:

\section{Remodelling AND AUtonomy of HEALTH CENTRES}

The health centre will serve as a local health authority, with management and financial autonomy, covering a population between 100,000 and 200,000 inhabitants; Family
Health Units (FHU) are the basis of care, allied health professions providing for all FHUs; rationalisation of acute and emergency care will be promoted.

\section{IMPLEMENTATION OF FAMILY HeALTH UNITS}

Through the creation of self-organised multi-professional teams, doctor's patient's lists are the basis for the practice population. FHUs have a maximum of eight GP's for 14,000 registered patients, there is a mixed payment system (capitation / salary / objectives), financial and professional incentives, and patients are expected to have a high access to care.

\section{Restructuring of Public Health Services}

There will be a shift from a regulatory role to epidemiological surveillance, geo-demographic organisation covering 100,000-200,000 inhabitants, and a local health observatory is envisaged.

\section{OTHER DIMENSIONS OF COMMUNITY INTERVENTION}

Reorganisation of community intervention, enhancement of home care, enhancement of palliative care, implementation of mobile units in selected situations and implementation of family support networks are planned.

\section{IMPLEMENTATION OF LOCAL HEALTH UNITS}

Enhancement of communication between health centres and hospitals and trial of new organisational models are planned.

\section{DeVelopment of human RESOURCES}

The admission of newly-graduated family physicians is to be facilitated, publicity for the new system is being designed to attract more nurses, mobility between practices is facilitated, part-time practice is allowed, activities such as tutoring, teaching and research are rewarded and other health professions will be brought into the health centres.

\section{Development of health INFORMATION SYSTEMS}

An integrated health information system covering all health centres and FHU's is under development, a data centre allowing access to information anywhere in the country will be established, a network for data sharing among health professionals and communication with patients will be created, and guidelines and specification for software developers are being implemented.

\section{Change and competency DEVELOPMENT}

Staff development will be promoted through the training for change management, continuing professional development of health professionals, involvement in pre-graduate education and postgraduate training.

The initial phase, and the one with the most public visibility, is the creation of Family Health Units (FHU), small multi-professional teams, composed of three to eight family doctors, an equal number of family nurses and receptionists, encompassing a population between 4,000 and 14,000 people. These teams will have technical, functional and organisational autonomy, and systems that will reward merit, and are sensitive to productivity, accessibility and quality.

The FHU's responsibility is to maintain and improve the health of the registered population, through general health care services, on a custom basis, with good accessibi- 
lity and continuity. They are health care services based on proximity, of small dimension, embedded in the community, of easy contact and friendly relation with its users.

To reach this goal it is essential in the next few years to make an effort to re-qualify the existing structures, the current network of health centres and health stations, to build new structures more adequate to the needs and closer to the people, but above all, to re-train the human resources in the current and future FHU's.

It is crucial to de-bureaucratise and modernise the procedures in health centres and to promote change towards systematic identification of processes that can be simplified to the benefit of the citizens, increasing the efficiency and better use of professionals' time and labour. More organisational and management flexibility is needed, more teamwork, more autonomy and accountability, a new contract and evaluation culture, and an information system that answers the needs.

The big challenges to the new health policy are influenced by matters like demographic change and the aging of populations, the growing complexity of health care, technological development, the increase in the citizens' expectations and financing problems, which influence not only the present, but also the future.

The potential for transformation and innovation in the Portuguese Health Services is still great. There is a need for permanent improvements in quality, with an aim to meeting the needs, expectations and the satisfaction of the population that uses them, as well as increasing the satisfaction of health professionals and the improving organisation and management. For that to occur, the needs and expectations of the recipients and the providers of health care should be considered.

To plan and lead the reform, a ministry board was created in directly responsible to the Ministry of $\mathrm{He}$ alth, the "Taskforce for the reform of Primary Health Care" (MCSP). Its mission was the guidance of the launching of the overall project, coordination and tracking of the reconfiguration strategy of the health centres and the implementation of the family health units.

By the end of September 2007 there were 199 proposals for FHUs involving 3,021 professionals, 1,066 family physicians, 1,094 nurses, and 861 receptionists, covering $1,885,585$ patients. The average list size is 1,769 . The average FHU has around 9,475 patients, 7 doctors and 20 professionals in total. Since the number of family physicians in Portugal is around 6,000 and the Portuguese population is roughly 10 600,000 , this means $17.8 \%$ of the total number of family physicians are involved in the program and $17.8 \%$ of the population is covered.

Most of the professionals that work in the health centres, as well as the citizens that use them, are aware of their problems and the reasons for the problems. They have experienced both progress and mistakes and setbacks. They have grown weary of the contradictions between the political discourse favourable to primary health care and the day-to-day practice. They are in the best position to contribute to the transformation of Portuguese Health Centres for the better.

There is also significant enthusiasm and optimism among some sectors of the profession. Nevertheless, there is a considerable concern caused by the frequent political changes in the leadership of the Portuguese
NHS that might jeopardize the course of the reform. In 1999, when the new EPS was created, a considerable number of family physicians invested substantial energy and time in organising group practices but faced delays and difficulties with the bureaucracy of the Health Ministry that slowed down the pace of the reform.

\section{CONCIUSION}

After twenty-five years of the existence of modern family medicine in Portugal, it is important to recall the whole course of a journey taken together with our colleagues and friends from other countries and from other cultures.

Indeed, in some countries family medicine has managed to achieve considerable success for several years, there is great trust in the services provided, the professionals receive high-quality specific training, they are used to developing and applying protocols and guidelines, they possess good academic departments of family medicine, they have achieved a relationship of respect and de facto equality with other specialties and have strong professional associations. The professionals are held in great esteem by the citizens, acknowledged by those in administration, are held in high consideration by other specialists and have great self-esteem, in addition to relatively high incomes.

Portuguese Family Medicine has always tried to observe and study the successes of colleagues abroad. Despite following a path of its own, the support and cultural influence of systems where family medicine enjoys great prestige and experience has been paramount, and without it many of the achievements 
of Portuguese family medicine would not have been possible.

In the future this joint path should be reinforced and perfected in order to allow improvement in the expectations of Portuguese family doctors and their patients.

\section{REFERENCES}

1. Bentes M, Dias CM, Sakellarides C, Bankauskaite V. Health care systems in transition: Portugal. Copenhagen: WHO Regional Office for Europe on behalf of the European Observatory on Health Systems and Policies; 2004. Available in: URL: http://www.euro.who.int/document/ e82937.pdf [accessed 23 Setember 2007].

2. Diogo C. The Reform of the NHS in Portugal. University of York Department of Management Studies Working Paper No. 5. Available in: URL: http://www.york.ac.uk/ management/research/workingpapers/ workingpaper5.pdf [accessed 23 Setember 2007].

3. Veiga TR, Guardado-Moreira MJ, Fernandes AA. Social changes and better health conditions of the Portuguese population 1974-2000. Available in: URL: http:// www.ep.liu.se/ej/hygiea/ra/027/paper.pdf [accessed 23 September 2007].

4. GP in Europe. Portugal, the Health Care System. UEMO. Available in: URL: http://www.uemo.org [accessed 23 Setember 2007].

5. European Observatory of Health Systems and Policies. Health Care Systems in Transition. HiT summary: Portugal; 2004.

6. Macinko J, Starfield B, Shi L. The contribution of primary care systems to health outcomes within Organization for Economic Cooperation and Development (OECD) countries, 1970-1998. Health Serv Res 2003, 38 (3): 831-65.

7. Guichard S. The reform of the health care system in Portugal. Economics Department Working Papers no. 405. Paris: OECD; 2004. Available in: URL: http:// www.olis.oecd.org/olis/2004doc.nsf/43bb 6130e5e86e5fc12569fa005d004c/d74128 d2c3009d51c1256f2a0079dee4/\$FILE/JT 00170911.PDF [accessed 23 September 2007].

8. Sousa JC. New worlds reaped from the world. In: Alves MV, Ramos V, editors. Da Memória (On Memory). Lisboa: MVA Invent / Pfizer / APMCG; 2003. p. 79-89.

9. WHO/UNICEF. Primary health care: report of the International Conference of Primary Care. Geneva: WHO; 1978.

10. Sakellarides C. De Alma-Ata a Harry Potter: um testemunho pessoal (From Alma-Ata to Harry Potter: a personal statement). Rev Port S Pública 2001; Volume Temático (2): 101-8.

11. Sousa JC, Sardinha AM, Perez y Sanchez J, Ribas MJ. Os cuidados de saúde primários e a medicina geral e familiar em Portugal (Primary Health Care and Family Medicine in Portugal). Rev Port S Pública 2001; Volume Temático (2): 63-74.

12. Associação Portuguesa dos Médicos de Clínica Geral, Direcção Nacional. Um futuro para a medicina de família em Portugal (A future for family medicine in Portugal). Lisboa: APMCG; 1991.

13. Horder J. General Practice / Family Medicine: Portugal and the World. In: Alves MV, Ramos V, editors. Da Vontade (On Will). Lisboa: MVA Invent / Pfizer / APMCG; 2004. p. 17-47.

14. Horder J. The Portuguese Association of General Practice 2004: a report for the Royal College of General Practitioners. Rev Port Clin Geral 2005; 21: 325-7.

15. Anker H, Borchgrevink CF. Primary health care in Portugal: reflections on a Portuguese-Norwegian development project. Fam Pract 1985 Sep; 2 (3): 59-64.

16. The Future General Practitioner. Report of a working party of the Royal College of General Practitioners. London: RCGP; 1972.

17. Ministério dos Assuntos Sociais. Portaria 357/80 de 28 de Junho (Law 357/80). Diário da República, 1980.

18. Ordem dos Médicos. Colégio de Clínica Geral. Um novo curriculum para uma nova especialidade (A new curriculum for a new specialty). Lisboa: Ordem dos Médicos; 1995.

19. Santos I, Jordão JG. Internato Complementar de Clínica Geral: situação actual e evolução (Vocational training in General Practice. present state and evolution). Educ Med 1997; 8 (2): 69-83.

20. Ramos V. State of art and a vision of the future. Primary Health Care in the European Union. Workshop on "Training Programme in Primary Health Care, the function of Training and Resource Health
Centres”. Hungary; 1997.

21. Santos I, Andrade I. Teachers and trainers in General Practice: attributes and learning areas. Lisboa: ICGZS; 1995.

22. Jordão JG. Academic family medicine and the University development of the discipline in Portugal. In: Alves MV, Ramos V, editors. Da Memória (On Memory). Lisboa: MVA Invent / Pfizer / APMCG; 2003. p. $69-78$.

23. OECD health data 2004. Paris: OECD; 2004.

24. Barriers and Facilitators to Enhancing Interdisciplinary Collaboration in Primary Health Care. Available in: URL: http://www.eicp-acis.ca/en/resources/pdfs/Barriers-and-Facilitators-toEnhancing-Interdisciplinary-Collaboration-in-Primary-Health-Care.pdf [Accessed 23 September 2007].

25. Enhancing Interdisciplinary Collaboration in Primary Health Care in Canada. Available in: URL: http://www.eicpacis.ca/en/resources/pdfs/Enhancing-Interdisciplinary-Collaboration-in-PrimaryHealth-Care-in-Canada.pdf [Accessed 23 September 2007].

26. World Health Organization. Preparing a health care workforce for the 21 st century: the challenge of chronic conditions. Available in: URL: http://www.who. int/chp/knowledge/publications/workforce_report.pdf [Accessed 23 September 2007].

27. Ministério da Saúde. 1998. Decreto-lei 117/98: Regime remuneratório experimental dos médicos da carreira de clínica geral que exerçam funções nos centros de saúde do Serviço Nacional de Saúde (Law 117/98: Experimental Payment System for General Practitioners Working in Primary Care Centers within the National Health Service). Diário da República 103: 1991-4.

28. Oliveira MD, Magone J, Pereira A. Nondecision making and inertia in Portuguese health policy. J Health Polit Policy Law 2005 Feb-Apr; 30 (1-2): 211-30.

29. Missão para os Cuidados de Saúde Primários. Linhas de Acção Prioritária para o Desenvolvimento dos Cuidados de Saúde Primários [Priorities for the development of primary health care]. Lisboa: MCSP; 2006. 